\title{
Corneal Involvement in Graves' Orbitopathy: An In Vivo Confocal Study
}

\author{
Edoardo Villani, ${ }^{1,2}$ Francesco Viola, ${ }^{1,2}$ Roberto Sala, ${ }^{1,2}$ Mario Salvi, ${ }^{1,3}$ Chiara Mapelli, ${ }^{1,2}$ \\ Nicola Currò, ${ }^{1,2}$ Guia Vannucchi, ${ }^{1,3}$ Paolo Beck-Peccoz, ${ }^{1,3}$ and Roberto Ratiglia ${ }^{1,2}$
}

Purpose. To study the clinical involvement of the ocular surface and the in vivo morphology of corneal cells and nerves, in patients affected by active and inactive Graves' orbitopathy (GO).

Methods. The study included 26 consecutive GO patients and 20 age- and sex-matched healthy control subjects. GO was diagnosed on the basis of the criteria of the European Group on Graves' Orbitopathy, and disease activity was evaluated by the Clinical Activity Score (CAS). Each participant underwent a full eye examination, including an evaluation of symptoms (Ocular Surface Disease Index score), tear break-up time, fluorescein and lissamine green staining, corneal apex sensitivity, and Schirmer's test. The corneal apex was examined by means of confocal microscopy to investigate the number and morphology of epithelial and stromal corneal cells and subbasal nerves.

RESULTS. Eleven (43\%) of the 26 patients had active GO. Oneway ANOVA with the least-significant difference (LSD) post hoc test revealed statistically significant differences between patients and controls in all the evaluated parameters, except corneal sensitivity and nerve reflectivity. Among the GO patients, the only significant difference observed in active compared with inactive disease was in the number of hyperreflective (activated) keratocytes $(P<0.001$, LSD). Corneal sensitivity correlated inversely with proptosis ( $P<0.001$, Spearman's test).

Conclusions. GO patients show clinical and confocal corneal alterations and signs and symptoms partially related to dry eye disease. The ocular surface inflammation in GO seems to be due to both the dry eye and the autoimmune orbitopathy. (Invest Ophthalmol Vis Sci. 2010;51:4574-4578) DOI: 10.1167/iovs. 10-5380

G raves' orbitopathy (GO), which in most cases is bilateral, is the most frequent extrathyroidal expression of Graves' disease. ${ }^{1}$ Its pathogenesis is thought to be autoimmune. ${ }^{2}$ The main risk factors include cigarette smoking, thyroid dysfunc-

From the ${ }^{1}$ Università degli Studi di Milano, Milan, Italy; the ${ }^{2}$ Department of Specialty Surgical Sciences, UO Oculistica Fondazione Ospedale Maggiore Policlinico, Mangiagalli e Regina Elena, IRCCS (Istituto Ricerca e Cura a Carattere Scientifico), Milan, Italy; and the ${ }^{3}$ Department of Medical Sciences, UO Endocrinologia Fondazione Ospedale Maggiore Policlinico, Mangiagalli e Regina Elena, IRCCS, Milan, Italy.

Presented at the annual meeting of the Association for Research in Vision and Ophthalmology, Fort Lauderdale, Florida, May 2009.

Submitted for publication February 12, 2010; revised March 17, 2010; accepted March 29, 2010.

Disclosure: E. Villani, None; F. Viola, None; R. Sala, None; $\mathbf{M}$. Salvi, None; C. Mapelli, None; N. Currò, None; G. Vannucchi, None; P. Beck-Peccoz, None; R. Ratiglia, None

Corresponding author: Edoardo Villani, Department of Specialistic Surgical Sciences, UO Oculistica Fondazione Ospedale Maggiore Policlinico, Mangiagalli e Regina Elena, IRCCS, Via Francesco Sforza 35, 20122 Milan, Italy; eddy.villani@tiscali.it. tion, and, sometimes, radioiodine therapy for Graves' hyperthyroidism. ${ }^{3}$

The clinical manifestations of GO reflect the increase in orbital volume, which may lead to higher intraorbital pressure, proptosis or exophthalmos, lid retraction, excessive corneal exposure, diplopia and/or strabismus, soft tissue changes with periorbital edema, conjunctival hyperemia and chemosis, nocturnal lagophthalmos, and the absence of Bell's phenomenon. ${ }^{1}$ The alterations of the ocular surface, resulting in both a hyposecretory and evaporative mechanism, modifies the tear film and leads to the development of dry eye syndrome. ${ }^{4}$

GO is also characterized by various inflammatory signs, including inflammatory infiltration of the orbital tissues, conjunctiva and caruncula, which are used to assess its activity by means of the Clinical Activity Score (CAS) ${ }^{5}$ and reflect the role of inflammation in the pathogenesis of dry eye. ${ }^{1,6}$

Confocal microscopy is a rapid and noninvasive technique that enables examination of the different layers of the cornea in vivo (cells and nerve fibers) and detection of possible alterations. Some of these have been recently interpreted as signs of inflammatory activity ${ }^{7}$ : The presence of Langerhans' cells in subepithelial infiltrates after epidemic keratoconjunctivitis has been shown to be related to immunologically mediated response, ${ }^{8}$ and epithelial dendritic cells have been reported to be modified in immune-mediated inflammation, such as graft rejection and recurrent herpetic keratitis. ${ }^{9}$ The number of beadlike formations (in herpetic keratitis and rheumatoid arthritis), ${ }^{10,11}$ and the number of activated keratocytes (in rheumatoid arthritis and Sjögren's syndrome $)^{11,12}$ have been interpreted as confocal signs of ocular surface disease activity.

The purpose of this study was to analyze clinical and confocal changes in the ocular surface of patients with active and inactive GO.

\section{Methods}

\section{Patients}

We consecutively recruited 26 patients ( 8 men and 18 women; mean age, $50.75 \pm 16.82$ years; range, 29-75), who had a diagnosis of GO based on the criteria of the European Group on Graves' Orbitopathy (EUGOGO) Consensus Statement. ${ }^{5,13}$ All the patients were recruited at the EUGOGO Center of the Fondazione IRCCS Ospedale Maggiore Policlinico, Mangiagalli e Regina Elena, Milan, Italy. GO activity was defined by using the CAS, ${ }^{14}$ which was assigned after each patient's examination by an ophthalmologist and endocrinologist. This score is based on four well-known classic signs of inflammation (pain, redness, swelling, and impaired function) and consists of 10 items. For each sign that is present, one point is given. Each sign has the same weight. The sum of these points is the CAS (range, $0-10){ }^{14}$

The control group consisted of 20 healthy subjects ( 7 men and 13 women; mean age, $51.20 \pm 18.20$ years; range, $27-76$ ) affiliated with our general clinic. 
TABLE 1. Clinical Data: Systemic Pathology and Orbitopathy

\begin{tabular}{lccr}
\hline & Inactive GO & Active GO & $\boldsymbol{P}^{*}$ \\
\hline GO & & & \\
$\quad$ Time since symptom onset, mo & $40.25 \pm 57.13$ & $16.81 \pm 8.24$ & 0.139 \\
$\quad$ Time since diagnosis, mo & $34.00 \pm 56.49$ & $14.27 \pm 9.26$ & 0.203 \\
CAS & $0.93 \pm 0.79$ & $3.72 \pm 0.90$ & $<0.001$ \\
Proptosis, mm & & & \\
$\quad$ RE & $21.56 \pm 3.88$ & $23.27 \pm 2.64$ & 0.196 \\
LE & $22.10 \pm 3.71$ & $23.04 \pm 2.74$ & 0.463 \\
TSH, mU/L & $1.11 \pm 1.14$ & $2.37 \pm 2.64$ & 0.166 \\
FT4, pmol/L & $3.95 \pm 3.71$ & $5.93 \pm 6.14$ & 0.369 \\
FT3, pmol/L & $4.39 \pm 3.35$ & $5.23 \pm 2.09$ & 0.455 \\
\hline
\end{tabular}

* $t$-Test.

Patients with lymphomas, AIDS, sarcoidosis, diabetes mellitus, or corneal dystrophy; those in treatment with systemic corneotoxic drugs or having local treatments with anti-glaucoma or steroidal or nonsteroidal anti-inflammatory drugs (NSAIDs); and those wearing contact lenses or having undergone ophthalmic surgery were excluded from the study.

All subjects gave their written informed consent for participation in the study, which was conducted in accordance with the guidelines of the Declaration of Helsinki and the Italian privacy law.

\section{Clinical Evaluation}

The medical history of each participant was carefully recorded.

Any subjective symptoms of a disease affecting the ocular surface and their impact on visual function were evaluated according to the standardized Ocular Surface Disease Index (OSDI), and the score was calculated. ${ }^{15}$

All subjects underwent a thorough ophthalmic examination of both eyes, including a biomicroscopic examination of the ocular adnexa and anterior segment. The cornea was stained with sodium fluorescein $1 \%$ collyrium, and the tear film break-up time (BUT) was quantified with cobalt blue light; corneal staining was assessed with the CLEK schema,${ }^{16}$ with the cornea divided into five areas. Subsequently, the conjunctiva was stained with lissamine green and analyzed with the CLEK schema ${ }^{16}$ in four areas. The staining scale was 0 to 4 , with 0.5 unit steps in each region. Photographs were used as examples of severity. Corneal sensitivity was evaluated with a Cochet-Bonnet nylonthread esthesiometer. Finally, Schirmer's test was performed after application of topical anesthesia (oxybuprocaine chloride $0.4 \%$ ).

\section{Confocal Microscopy}

Image Acquisition. After a drop of the anesthetic had been instilled in the lower conjunctival fornix, the corneal apex was examined by confocal microscope (Confoscan 3.0; Nidek Technologies, Vigonza, Italy) equipped with a standard $40 \times$ objective. During the examination, the objective lens of the microscope was covered with gel (hydroxypropylmethyl cellulose 0.3\%, Carbopol 980; Noveon Europe, Brussels, Belgium, or Dequest 2060S; Solutia, St. Louis, MO) and never came into direct contact with the corneal surface. A drop of antibiotic (ofloxacin $0.3 \%$ ) was instilled in the lower conjunctival fornix at the end of each examination, and the eye was re-examined by means of a slit lamp to verify the integrity of the corneal surface.

The full thickness of the cornea was scanned automatically for 2 to 3 minutes. Each scan recorded $350 z$-axis images separated by a distance of $1.5 \mu \mathrm{m}$, making two to four complete passages from the lacrimal film to the anterior chamber. Each frame covered an area of approximately $440 \times 330 \mu \mathrm{m}$.

Image Analysis. $Z$-scan curves (graphs showing the depth coordinate on the $z$-axis and the level of reflectivity on the $y$-axis) ${ }^{17}$ were used to select the images relating to the superficial and basal epithelium, the anterior and posterior stroma, and Bowman's membrane or the subbasal epithelium. This method made it possible to evaluate the cell density of the superficial and basal epithelium, and the apparent cell density of the anterior and posterior stroma, concentrating particularly on the number of highly reflective (activated) keratocytes.

Cell density was determined with a manual cell counting procedure in the software, considering the entire area marked as available. Any cells partially contained in the analyzed area were only counted along the right and lower margins. The results are expressed as the number of cells per square millimeter.

The cell densities, obtained by a second independent investigator, were used to calculate the $K$-value to assess interobserver agreement.

The image of the subbasal plexus containing the largest number of recognizable nerve fibers was selected for each scan. Corneal innervation was studied by considering the number of nerves, the degree of tortuosity and fiber reflectivity graded 0 to 4 after comparison with the reference images, as suggested by Oliveira-Soto and Efron. ${ }^{18}$ The number of beadlike formations per $100 \mu \mathrm{m}$ of nerve fiber was counted as described by Benitez del Castillo et al. ${ }^{19}$

TABLE 2. Clinical Data for the Ocular Surface

\begin{tabular}{|c|c|c|c|c|}
\hline & Inactive GO & Active GO & Control Subjects & $\boldsymbol{P}^{*}$ \\
\hline Age, $y$ & $46.26 \pm 10.51$ & $56.63 \pm 9.98$ & $51.20 \pm 18.20$ & NS \\
\hline OSDI score & $22.01 \pm 17.39$ & $29.13 \pm 12.48$ & $0.53 \pm 0.54$ & $<0.001 \dagger$ \\
\hline BUT, s & $6.64 \pm 2.23$ & $6.45 \pm 3.07$ & $10.36 \pm 1.85$ & $<0.001 \ddagger$ \\
\hline Fluorescein staining, total score & $1.14 \pm 1.16$ & $0.90 \pm 0.30$ & & NS \\
\hline Lissamine green staining, total score & $0.71 \pm 0.61$ & $1.09 \pm 1.22$ & & NS \\
\hline Corneal sensitivity & $5.67 \pm 0.42$ & $5.43 \pm 0.822$ & $5.85 \pm 0.39$ & 0.12 \\
\hline Schirmer's test, mm & $9.84 \pm 2.96$ & $9.54 \pm 2.91$ & $15.03 \pm 3.51$ & $<0.001 \S$ \\
\hline
\end{tabular}

\footnotetext{
* ANOVA.
}

† Inactive GO, active GO vs. control; $P<0.001$, LSD. Inactive GO vs. active GO; $P=0.13$, LSD.

‡ Inactive GO, active GO vs. control; $P<0.001$, LSD. Inactive GO vs. active GO; $P=0.84$, LSD.

$\S$ Inactive GO, active GO vs. control; $P<0.001$, LSD. Inactive GO vs. active GO; $P=0.821$, LSD. 


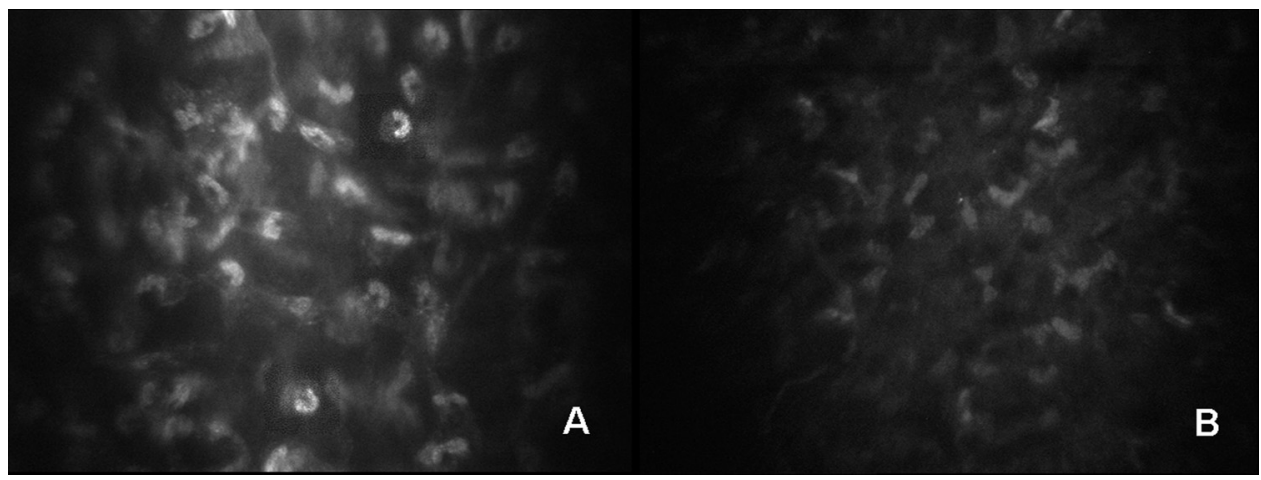

Figure 1. Confocal stromal images showing several activated keratocytes in a GO patient (A) and no activated keratocytes in a control subject (B).

\section{Statistical Analysis}

The data for the worse eye of each subject-that is, the eye with the highest fluorescein staining score-were included in the analysis. In the case of equal scores for the two eyes, the sequential discriminating criteria were the lower Schirmer's test score and the lower BUT score.

All the data are expressed as the mean $\pm \mathrm{SD}$. The normal distribution of the variables was verified using the Kolmogorov-Smirnov test. One-way ANOVA with the LSD post hoc test was used to compare the mean scores of the control subjects and patients with active or inactive GO and the $t$-test for independent samples to compare the GO clinical data (active GO versus inactive GO). The correlations between the variables were evaluated by using Spearman's index of linear correlation. $P<0.05$ was considered significant (SPSS for Windows, ver. 9.0; SPSS Sciences, Chicago, IL).

\section{Results}

\section{Patients' Clinical Data}

Eleven patients had active GO (43\%; 2 men and 9 women; mean age, $56.63 \pm 9.98$ years; range, 42-75), and 15 had inactive GO (57\%; 6 men and 9 women; mean age, $46.26 \pm$ 10.51 years; range, 29-67). CAS was significantly higher in active compared with inactive GO $(P<0.001, t$-test $)$; there were no other significant differences in the systemic clinical data in patients with active versus inactive GO (Table 1).

\section{Clinical Data: Ocular Surface}

The OSDI score was significantly higher in GO patients than in the control subjects, and BUT and Schirmer's test values were significantly reduced $(P<0.001$, LSD). No differences were found between the two GO groups (Table 2).

\section{Confocal Microscopy Data}

Cell Density. Cell density in the different layers was significantly different between the GO patients and the control subjects (superficial epithelium and anterior stroma: $P<$ 0.001, LSD; basal epithelium and posterior stroma: $P<0.05$, LSD), but there were no significant differences between the patients with active or inactive GO. However, the density of activated keratocytes (Fig. 1) was significantly different between the patient groups ( $P<0.001$, LSD; Table 3$)$.

There was substantial agreement between the two investigators in relation to all these variables: $K=0.75$ (superficial epithelium), 0.96 (basal epithelium), 0.78 (anterior stroma), 0.80 (posterior stroma), and 0.66 (activated keratocytes).

Innervation Data. A comparison of GO patients with controls showed a lower number of nerve fibers and an increased degree of tortuosity and number of beadlike formations (Fig. 2; $P<0.001$, LSD; Table 4).

\section{Correlations}

There were positive correlations between FT3 and FT4 levels and disease activity $(P<0.05$, Spearman) and between the onset of symptoms and diagnosis and the degree of proptosis $(P<0.05$, Spearman $)$. There was a significant inverse correlation between sensitivity at the corneal apex and proptosis $(P<$ 0.001 , Spearman). No significant correlations were found between confocal and clinical data.

\section{Discussion}

GO is the most frequent extrathyroidal expression of Graves' disease and has various ocular manifestations. ${ }^{1}$ Its onset apparently peaks bimodally in the fifth and seventh decades of life, but eye disease may occur at any age. ${ }^{20}$

The natural history of GO is not completely understood, but it is generally agreed that it has an initial active phase characterized by the progressive exacerbation of ocular manifestations and then tends to remit spontaneously, although the remission is invariably partial. ${ }^{21}$

TABLE 3. Cell Density Data from Confocal Microscopy

\begin{tabular}{|c|c|c|c|c|}
\hline & Inactive GO & Active GO & Control Subjects & $\boldsymbol{P}^{*}$ \\
\hline Superficial epithelium, cells $/ \mathrm{mm}^{2}$ & $1033.20 \pm 168.30$ & $1011.36 \pm 199.36$ & $1517.15 \pm 130.65$ & $<0.001 \dagger$ \\
\hline Basal epithelium, cells $/ \mathrm{mm}^{2}$ & $5816.26 \pm 209.69$ & $5806.81 \pm 240.09$ & $5600.95 \pm 235.94$ & $<0.05 \ddagger$ \\
\hline Anterior stroma, cells $/ \mathrm{mm}^{2}$ & $1189.46 \pm 89.24$ & $1215.81 \pm 88.71$ & $971.15 \pm 103.56$ & $<0.001 \S$ \\
\hline Posterior stroma, cells $/ \mathrm{mm}^{2}$ & $809.33 \pm 120.01$ & $787.72 \pm 116.85$ & $719.10 \pm 78.42$ & $<0.05 \|$ \\
\hline Activated keratocytes, cells/frame & $3.41 \pm 1.49$ & $6.04 \pm 2.93$ & $0.42 \pm 0.73$ & $<0.0019$ \\
\hline
\end{tabular}

\footnotetext{
* ANOVA.

† Inactive GO, active GO vs. control; $P<0.001$, LSD. Inactive GO vs. active GO; $P=0.735, \mathrm{LSD}$.

‡ Inactive GO, active GO vs. control; $P<0.05$, LSD. Inactive GO vs. active GO; $P=0.918$, LSD.

$\int$ Inactive GO, active GO vs. control; $P<0.001$, LSD. Inactive GO vs. active GO; $P=0.492$, LSD

$\|$ Inactive GO vs. active GO; $P=0.599$, LSD. Inactive GO vs. control; $P<0.05$, LSD. Active GO vs. control; $P=0.083$, LSD.

II Inactive GO, active GO vs. control; $P<0.001$, LSD. Inactive GO vs. active GO; $P<0.001$, LSD.
} 


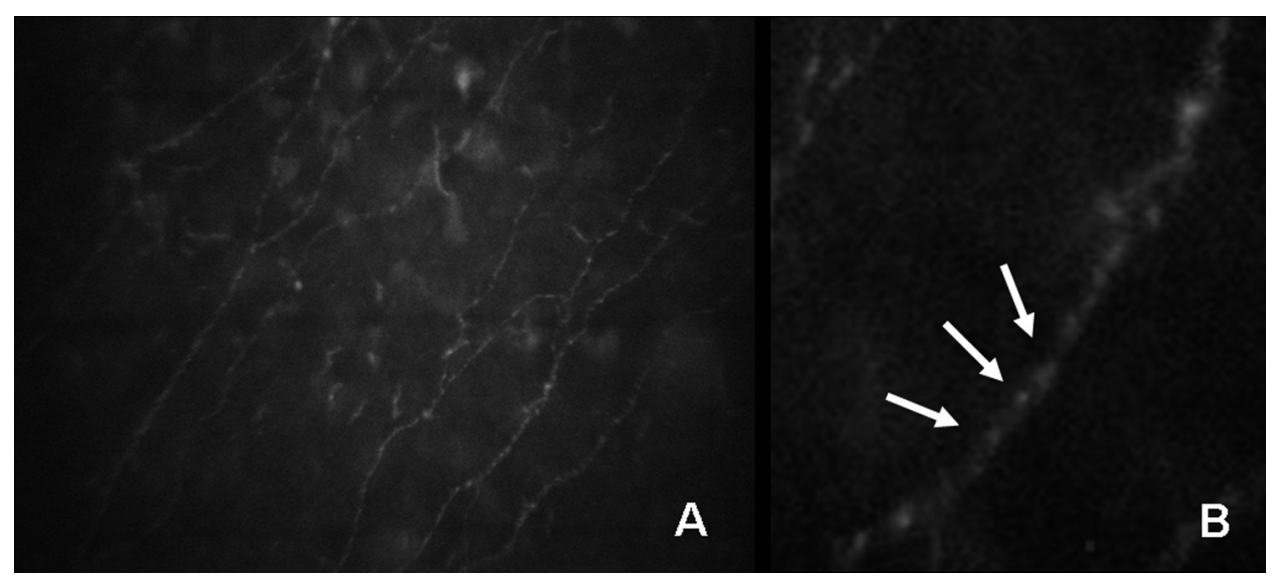

FIGURE 2. Confocal image showing subbasal nerve fibers (A), with evident beadlike formations (B, arrows).

The degree of proptosis in our patients was greater than that observed in previous studies of thyroid orbitopathy. ${ }^{22-25}$ It correlated with the onset of symptoms and diagnosis and was probably due to greater severity.

All GO patients had signs and symptoms characteristic of dry eye. ${ }^{26}$ The OSDI scores of the GO patients and control subjects were significantly different, but the slight difference between the patients with active or inactive disease was not statistically significant. There was no significant correlation between the OSDI and Schirmer's type 1 test, tear BUT, or fluorescein or lissamine green staining, which is not surprising in patients with dry eye. ${ }^{27}$

The Schirmer test results were significantly different between the patients and controls but, although the patients' results $(6-13 \mathrm{~mm})$ suggested mild lacrimal hyposecretion, ${ }^{24}$ they did not explain the entire ocular surface disease. Tear BUT was significantly shorter in the patients, suggesting an unstable tear film. This finding was consistent with those of patients with long-standing $\mathrm{GO}^{23,25}$ but was higher than those reported in patients with active thyroid-associated orbitopathy. ${ }^{24,28}$ Tear film instability is considered to be one of the core mechanisms involved in dry eye. ${ }^{4}$

Ocular surface fluorescein and lissamine green staining was surprisingly poor. The results were similar only to the data on corneal staining reported by Khurana et al., ${ }^{23}$ whereas most research groups have reported greater corneal and conjunctival staining, ${ }^{22,24,28}$ including Gupta et al., ${ }^{29}$ who reported that fluorescein staining of the cornea is the most common finding due to ocular surface inflammation in occult thyroid eye disease. However, a fluorescein staining score of 1 is the most frequent in $\mathrm{GO},{ }^{30}$ and higher scores have been recorded in only a very small samples of patients. ${ }^{30,31}$

The corneal sensitivity was only partially reduced in patients compared with control subjects. The difference was not statistically significant, and the reduction was not as great as in other types of dry eye. ${ }^{11,12,32}$ This result may explain the mild lacrimal hyposecretion revealed by Schirmer's test, which may be caused by both active inflammation and stimulation of lacrimal TSH receptors by Graves autoantibodies demonstrated by Eckstein et al. ${ }^{24}$

The dry eye associated with GO therefore seems to be mainly evaporative, due to increased corneal exposure. According to Gupta et al., ${ }^{29}$ patients with GO do not generally show poor aqueous production but, based on our Schirmer test results and the presence of inflammation, we believe that a hyposecretory component cannot be excluded and may contribute to both GO and dry eye syndrome, as also suggested by Eckstein et al. ${ }^{24}$

Over the past few years, confocal microscopy has provided new opportunities of studying the in vivo structure of the cornea. As in other recent studies, ${ }^{11,12,19}$ we used it to make a quantitative evaluation of corneal alterations, a major evolution in the application of this technology. The observed morphologic changes are similar to those recently described in various types of dry eye. ${ }^{11,12,19}$

The significant reduction in surface epithelial cell density in our GO patients was probably due to the damaged ocular surface caused by typical exophthalmos, lid retraction, nocturnal lagophthalmos, and the absence of Bell's phenomenon. On the contrary, the increased number of basal epithelium cells in GO patients may be caused by the proliferative stimulus induced in dry eye by superficial desiccating stress, as was demonstrated by Fabiani et al. ${ }^{33}$

The increased stromal cell density may be the result of the inflammatory process itself, which involves the ocular surface and causes a synchronous increase in stromal apoptotic phenomena, proteolytic activity, and proliferating stimuli. ${ }^{12}$

As previously observed by Tuominen, ${ }^{34}$ Villani et al., ${ }^{11}$ and Benitez del Castillo ${ }^{19}$ in different studies, the increased stromal cell density was accompanied by hyperreflective, activated keratocytes. These may be regarded as inflammatory cells that are in a specific stage of metabolic activation induced by proinflammatory cytokines such as IL-1 and $-6^{11}$ and are capa-

TABLE 4. Innervation: Confocal Microscopy

\begin{tabular}{|c|c|c|c|c|}
\hline & Inactive GO & Active GO & Control Subjects & $\boldsymbol{P}^{*}$ \\
\hline Nerves, $n$ & $3.29 \pm 1.13$ & $3.28 \pm 1.21$ & $5.04 \pm 0.83$ & $<0.001 \dagger$ \\
\hline Tortuosity & $2.49 \pm 0.91$ & $2.58 \pm 0.96$ & $1.33 \pm 0.64$ & $<0.001 \ddagger$ \\
\hline Reflectivity & $1.73 \pm 1.27$ & $2.60 \pm 1.40$ & $2.02 \pm 1.02$ & $0.194^{\circ}$ \\
\hline Beadlike formations, $n$ & $311.25 \pm 67.89$ & $345.61 \pm 65.24$ & $201.55 \pm 70.28$ & $<0.001 \S$ \\
\hline
\end{tabular}

\footnotetext{
* ANOVA.

† Inactive GO, active GO vs. control; $P<0.001$, LSD. Inactive GO vs. active GO; $P=0.976$, LSD

‡ Inactive GO, active GO vs. control; $P<0.001$, LSD. Inactive GO vs. active GO; $P=0.78$, LSD.

』 Inactive GO, active GO vs. control; $P<0.001$, LSD. Inactive GO vs. active GO; $P=0.212$, LSD.
} 
ble of producing proinflammatory cytokines such as nerve growth factor. ${ }^{12}$

Another largely reported confocal sign of inflammation is the number of dendritic cells. ${ }^{9}$ These cells, which may be interpreted as antigen-presenting cells, are clearly detectable by laser scanning confocal microscopy. In our experience, the white-light confocal microscope used in this study is more effective for analyzing the stroma but less useful for studying the subbasal dendritic cells. Therefore, we preferred not to use this variable to quantify inflammation.

It is important to note that the number of activated keratocytes was the only sign that was found to be significantly different when GO patients were compared to control subjects and also when active GO was compared to inactive GO. This result suggests that patients with active GO not only show more diffuse inflammation at the level of the ocular adnexa (as quantified by CAS), but are also more affected by corneal inflammation.

In GO patients, the number of nerves was reduced, and the tortuosity of the nerve fiber and the number of beadlike formations were increased, as was found in other studies of dry eye. ${ }^{11,19,35}$ This sign may not be specific for dry eye, but an indication of metabolic activation of the plexus ${ }^{35}$ related to the systemic disease (the NGF secreted by keratocytes makes a substantial contribution to this activation).

In conclusion, confocal microscopy is extremely useful for quantitatively evaluating ocular surface inflammation. It also provides a very interesting opportunity for future studies of ocular surface disease activity that integrate traditional clinical data (such as CAS) and new, rapid, and noninvasive methods of investigating in vivo tissue histology.

The ocular surface of GO patients seems to be simultaneously affected by two inflammatory processes: one secondary to dry eye and the other related to the systemic disease itself.

\section{References}

1. Prabhakar BS, Bahn RS, Smith TJ. Current perspective on the pathogenesis of Graves' disease and ophthalmopathy. Endocr Rev. 2003;24:802-835.

2. DeGroot LJ, Quintans J. The causes of autoimmune thyroid disease. Endocr Rev. 1989;10:537-562.

3. Wiersinga WM, Bartalena L. Epidemiology and prevention of Graves' ophthalmopathy. Thyroid. 2002;12:855-860.

4. Report of the Dry Eye WorkShop. Ocul Surf. 2007;5:65-204.

5. Bartalena L, Baldeschi L, Dickinson AJ, et al. Consensus statement of the European Group on Graves' orbitopathy (EUGOGO) on management of GO. Eur J Endocrinol. 2008;158:273-285.

6. Ajjan RA, Weetman AP. New understanding of the role of cytokines in the pathogenesis of Graves' ophthalmopathy. $J$ Endocrinol Invest. 2004;27:237-245.

7. Chiou AG, Kaufman SC, Kaufman HE, Beuerman RW. Clinical corneal confocal microscopy. Surv Opbthalmol. 2006;51:482500 .

8. Alsuhaibani AH, Sutphin JE, Wagoner MD. Confocal microscopy of subepithelial infiltrates occurring after epidemic keratoconjunctivitis. Cornea. 2006;25:778-780.

9. Mastropasqua L, Nubile M, Lanzini M, et al. Epithelial dendritic cell distribution in normal and inflamed human cornea: in vivo confocal microscopy study. Am J Ophthalmol. 2006;142:736-744.

10. Martone $G$, Alegente $M$, Balestrazzi A, et al. In vivo confocal microscopy in bilateral herpetic keratitis: a case report. Eur $J$ Opbthalmol. 2008;18:994-997.

11. Villani E, Galimberti D, Viola F, Mapelli C, Del Papa N, Ratiglia R. Corneal involvement in rheumatoid arthritis: an in vivo confocal study. Invest Ophthalmol Vis Sci. 2008;49:560-564.

12. Villani E, Galimberti D, Viola F, Mapelli C, Ratiglia R. The cornea in Sjögren's syndrome: an in vivo confocal study. Invest Ophthalmol Vis Sci. 2007;48:2017-2022.
13. Wiersinga WM, Perros P, Kahaly GJ, et al. Clinical assessment of patients with Graves' orbitopathy: the European Group on Graves' Orbitopathy recommendations to generalists, specialists and clinical researchers. Eur J Endocrinol. 2006;155:387-389.

14. Mourits MP, Prummel MF, Wiersinga M, Koornneef L. Clinical activity score as a guide in the management of patients with Graves' ophthalmopathy. Clin Endocrinol. 1997;47:9-14.

15. Schiffman RM, Christianson MD, Jacobsen G, Hirsch JD, Reis BL. Reliability and validity of the Ocular Surface Disease Index. Arch Opbthalmol. 2000;118:615-621.

16. Barr JT, Schechtman KB, Flink BA, et al. Corneal scarring in the Collaborative Longitudinal Evaluation of Keratoconus (CLEK) Study: baseline prevalence and repeatability of detection. Cornea. 1999; 18:34- 46 .

17. Mastropasqua L, Nubile M. Basic principles of confocal microscopy of the cornea. In: Confocal Microscopy of the Cornea. Thorofare, NJ: SLACK, Inc.; 2002:3-4.

18. Oliveira-Soto L, Efron N. Morphology of corneal nerves using confocal microscopy. Cornea. 2001;20:374-384.

19. Benitez del Castillo JM, Wasfy MAS, Fernandez C, Garcia-Sanchez J. An in vivo confocal masked study on corneal epithelium and subbasal nerves in patients with dry eye. Invest Ophthalmol Vis Sci. 2004; 45:3030-3035.

20. Bartley GB. The epidemiological characteristics and clinical course of ophthalmopathy associated with autoimmune thyroid disease in Olmsted County, Minnesota. Trans Am Opbthalmol Soc. 1994;92: 477-588.

21. Wiersinga WM, Prummel MF. Graves' ophthalmopathy: a rational approach to treatment. Trends Endocrinol Metab. 2002;13:280287.

22. Gilbard JP, Farris RL. Ocular surface drying and tear film osmolarity in thyroid eye disease. Acta Opbthalmol (Copenb). 1983;61:108116.

23. Khurana AK, Sunder S, Ahluwalia BK, Malhotra KC. Tear film profile in Graves' ophthalmopathy. Acta Opbthalmol (Copenb). 1992; $70: 346-349$.

24. Eckstein AK, Finkenrath A, Heiligenhaus A, et al. Dry eye syndrome in thyroid-associated ophthalmopathy: lacrimal expression of TSH receptor suggests involvement of TSHR-specific autoantibodies. Acta Ophthalmol Scand. 2004;82:291-297.

25. Iskeleli G, Karakoc Y, Abdula A. Tear film osmolarity in patients with thyroid ophthalmopathy. Jpn J Opbthalmol. 2008;52:323326.

26. Perry HD, Donnenfeld ED. Dry eye diagnosis and management in 2004. Curr Opin Ophthalmol. 2004;15:299-304.

27. Nichols KK, Nichols JJ, Mitchell GL. The lack of association between signs and symptoms in patients with dry eye disease. Cornea. 2004;23:762-770.

28. Nowak M, Marek B, Kos-Kuda B, Kajdaniuk D, Siemiñska L. Tear film profile in patients with active thyroid orbitopathy. Klin Oczna. 2005;107:479-482.

29. Gupta A, Sadeghi BA, Akpek EK. Occult thyroid eye disease in patients presenting with dry eye symptoms. Am J Ophthalmol. 2009;147:919-923.

30. Brasil1 MV, Brasil OF, Vieira RP, Vaisman M, Amaral Filho OM. Tear film analysis and its relation with palpebral fissure height and exophthalmos in Graves' ophthalmopathy (in Portuguese). Arq Bras Oftalmol. 2005;68:615-618.

31. Coll J, Anglada J, Tomas S, et al. High prevalence of subclinical Sjögren's syndrome features in patients with autoimmune thyroid disease. J Rbeumatol. 1997;24:1719-1724.

32. $\mathrm{Xu} \mathrm{KP}$, Yagi $\mathrm{Y}$, Tsubota $\mathrm{K}$. Decrease in corneal sensitivity and change in tear function in dry eye. Cornea. 1996;15:235-239.

33. Fabiani C, Barabino S, Rashid S, Dana MR. Corneal epithelial proliferation and thickness in a mouse model of dry eye. Exp Eye Res. 2009;89:166-171.

34. Tuominen IS, Konttinen YT, Vesaluoma MH, Moilanen JA, Helinto M, Tervo TM. Corneal innervation and morphology in primary Sjögren's syndrome. Invest Opbthalmol Vis Sci. 2003;44:25452549.

35. Zhang M, Chen I, Luo L, Xiao Q, Sun M, Liu Z. Altered corneal nerves in aqueous tear deficiency viewed by in vivo confocal microscopy. Cornea. 2005;24:818-824. 